\title{
Interleukin 31 mediates MAP kinase and STAT1/3 activation in intestinal epithelial cells and its expression is upregulated in inflammatory bowel disease
}

\author{
Julia Dambacher, Florian Beigel, Julia Seiderer, Dirk Haller, Burkhard Göke, Christoph \\ J Auernhammer, Stephan Brand
}

See end of article for authors' affiliations

Correspondence to:

Dr Stephan Brand, University Hospital Munich-

Grosshadern, Department of Medicine II, University of Munich, Marchioninistrasse 15, 81377 Munich

Germany; stephan.brand@ med.uni-muenchen.de

Revised 21 March 2007 Accepted 10 April 2007 Published Online First 20 April 2007
Background/aim: Interleukin 31 (IL31), primarily expressed in activated lymphocytes, signals through a heterodimeric receptor complex consisting of the IL31 receptor alpha (IL31R $\alpha$ ) and the oncostatin M receptor (OSMR). The aim of this study was to analyse IL31 receptor expression, signal transduction, and specific biological functions of this cytokine system in intestinal inflammation.

Methods: Expression studies were performed by RT-PCR, quantitative PCR, western blotting, and immunohistochemistry. Signal transduction was analysed by western blotting. Cell proliferation was measured by MTS assays, cell migration by restitution assays.

Results: Colorectal cancer derived intestinal epithelial cell (IEC) lines express both IL31 receptor subunits, while their expression in unstimulated primary murine IEC was low. LPS and the proinflammatory cytokines TNF- $\alpha$, IL $1 \beta$, IFN- $\gamma$, and sodium butyrate stimulation increased IL31, IL31R $\alpha$, and OSMR mRNA expression, while IL31 itself enhanced IL8 expression in IEC. IL31 mediates ERK-1/2, Akt, STATI, and STAT3 activation in IEC resulting in enhanced IEC migration. However, at low cell density, IL31 had significant antiproliferative capacities $(p<0.005)$. IL31 mRNA expression was not increased in the TNF $\triangle$ ARE mouse model of ileitis but in inflamed colonic lesions compared to non-inflamed tissue in patients with Crohn's disease (CD; average 2.4fold increase) and in patients with ulcerative colitis (UC; average 2.6-fold increase) and correlated with the IL8 expression in these lesions ( $r=0.564$ for $C D ; r=0.650$ for UC; total number of biopsies analysed: $n=88$ ). Conclusion: IEC express the functional IL31 receptor complex. IL31 modulates cell proliferation and migration suggesting a role in the regulation of intestinal barrier function particularly in intestinal inflammation.
$\mathrm{R}$ ecently, interleukin 31 (IL31) has been identified as a four helix bundle cytokine that is produced by activated T cells, particularly by T helper type 2 cells. ${ }^{1}$ IL 31 is closely related to the IL6-type cytokines oncostatin M (OSM), leukaemia inhibitory factor (LIF), and cardiotrophin-1 and signals through a heterodimeric receptor complex composed of the IL31 receptor alpha (IL31R $\alpha)$ and the oncostatin $M$ receptor (OSMR). ${ }^{1}$ IL31R $\alpha$ is identical to the gpl30-like receptor (GPL). ${ }^{1}$ At least four splice variants of IL31R $\alpha$ have been described. ${ }^{1}$ The short isoform of IL31R $\alpha$ exerts a strong inhibitory effect on the signalling of IL31 and behaves as a dominant negative receptor. ${ }^{2}$ The tyrosine residues 652 and 721 in the cytoplasmic region of the long isoform of IL31R $\alpha / G P L(745)$ have been identified as the major STAT5 and STAT3 activating sites, respectively. ${ }^{3}$ IL31R $\alpha$ has sequence and domain homologies with gp130, LIFR, and granulocyte colony stimulating factor receptor. ${ }^{1}$ Expression of IL31R $\alpha$ and OSMR mRNA has been shown to be induced in activated monocytes while epithelial cells express mRNA for both receptors constitutively. ${ }^{1}$ Activation of the receptor complex resulted in recruitment of Jak1, Jak2, STAT1, STAT3, STAT5, and PI3 kinase signalling pathways in glioblastoma and melanoma tumour cells, and lung epithelial cells. ${ }^{2}$ While IL31 is the only ligand for the IL $31 \mathrm{R} \alpha$ receptor subunit identified so far, the OSMR subunit is also used by OSM, which signals through a receptor complex consisting of OSMR and gpl30.5

So far, biological functions of this novel cytokine were mainly analysed in certain skin diseases such as atopic dermatitis. ${ }^{6}$ Transgenic mice overexpressing IL31 develop a skin disorder characterised by severe pruritus, alopecia, and skin lesions suggesting a role for IL31 in skin inflammation. ${ }^{1}$ A similar phenotype was induced after subcutaneous delivery of recombinant IL31 in mice. ${ }^{1}$ Peripheral lymph nodes from IL31 transgenic mice and IL31 protein treated mice were enlarged compared to non-treated and non-transgenic mice. ${ }^{1}$ In humans, IL31 was significantly overexpressed in pruritic atopic skin lesions. ${ }^{7}$ In vivo, staphylococcal superantigen rapidly induced IL31 expression in atopic individuals. ${ }^{7}$ Comparisons between skin from patients with atopic dermatitis and healthy skin showed high IL31R $\alpha$ expression on epidermal keratinocytes and increased IL31 expression in infiltrating cells in skin samples taken from atopic patients. ${ }^{8}$ Moreover, an increased IL31R $\alpha$ expression in diseased tissues derived from an animal model of airway hypersensitivity has been described.

While the role of IL31 in skin inflammation is well characterised, ${ }^{1-10}$ its biological properties in other tissues are largely unknown. Although IL31 expression has been described in the small intestine and colon, ${ }^{1}$ the expression of the IL31

Abbreviations: $C D$, Crohn's disease; ERK, extracellular signal regulated kinase; ELISA, enzyme linked immunosorbent assay; FCS, fetal calf serum; GAPDH, glyceraldehyde-3-phosphate dehydrogenase; GPL, gp130-like receptor; IBD, inflammatory bowel disease; IEC, intestinal epithelial cell; IFN, interferon; IL, interleukin; IL31R $\alpha$, interleukin 31 receptor alpha; LIF, leukaemia inhibitory factor; LIFR, leukaemia inhibitory factor receptor; LPS, lipopolysaccharide; MAP kinase, mitogen activated protein kinase; MCMV, murine cyłomegalovirus; MEK, mitogen activated protein kinase kinase; MTS, 3-(4,5-dimethylthiazol-2-yl)-5-(3-carboxymethoxyphenyl)-2-(4sulfophenyl)-2H-tetrazolium; OSM, oncostatin $M$; OSMR, oncostatin $M$ receptor; PCR, polymerase chain reaction; PI, phosphatidyl-inositol; RTPCR, reverse transcriptase polymerase chain reaction; SOCS, suppressor of cytokine signalling; STAT, signal transducer and activator of transcription; $\mathrm{TNF}-\alpha$, tumour necrosis factor alpha; UC, ulcerative colitis 
receptor complex has not been demonstrated in intestinal epithelial cells (IEC) so far. Based on the expression of IL31R $\alpha$ in epithelial tissues such as skin and lung epithelium and similarities between skin and gut demonstrated by us for other STAT inducing cytokines such as IL22, ${ }^{11}$ we analysed the IL31 receptor expression in the intestinal epithelium and characterised its signal transduction and its specific biological functions in IEC, particularly in intestinal inflammation.

\section{MATERIALS AND METHODS Reagents}

Polyclonal antibodies to phosphorylated extracellular signal regulated kinase (ERK) 1/2 (Thr183/Tyr185) and phospho-Akt (Ser473) were purchased from Cell Signaling (Beverly, MA, USA). Anti-ERK-1/2 and anti-Akt antibodies were also from Cell Signaling. Phospho-STATl antibody was from BD Transduction Laboratories (Franklin Lakes, NY, USA), phospho-STAT3 antibody from Upstate Biotechnology (Lake Placid, NY, USA), and antibodies against STATI and STAT3 were from Santa Cruz Biotechnology (Santa Cruz, CA, USA). Horseradish peroxidase conjugated secondary antibodies to mouse or rabbit IgG and chemiluminescent substrate (SuperSignal West Dura Extended Duration Substrate) were from Pierce (Rockford, IL, USA). Human IL31R $\alpha$ antibody was from R\&D Systems (Minneapolis, MN, USA). Recombinant human IL31, tumour necrosis factor (TNF)- $\alpha$, ILl $\beta$, and interferon (IFN)- $\gamma$ were obtained from R\&D Systems (Minneapolis, MN, USA). Lipopolysaccharide (LPS) from Escherichia coli (O26:B6) prepared by phenol extraction was purchased from Sigma ( $\mathrm{St}$ Louis, MO, USA) and prepared as dispersed sonicate in endotoxin-free water (Life Technologies, Rockville, MD, USA) before diluting to final concentration in supplemented media.

\section{Cell culture}

The human colorectal cancer derived IEC lines HT-29, SW480, HCT1 16, T84, CaCo-2, and DLD-1 were obtained from American Type Culture Collection (Rockville, MD, USA). Cells were grown in Dulbecco's modified Eagle medium (Gibco BRL/Life Technologies, Gaithersburg, MD, USA) with $100 \mathrm{IU} / \mathrm{ml}$ penicillin, $100 \mu \mathrm{g} / \mathrm{ml}$ streptomycin, and $10 \%$ heat inactivated fetal calf serum (PAA, Pasching, Austria) in a humidified $5 \% \mathrm{CO}_{2}$ atmosphere at $37^{\circ} \mathrm{C}$. For signal transduction experiments with IL31, cells were starved overnight in serum-free medium.

\section{Reverse transcriptase polymerase chain reaction}

Reverse transcriptase polymerase chain reaction (RT-PCR) was performed as previously described. ${ }^{12}$ Briefly, total RNA was

Table 1 Primers used for PCR analysis

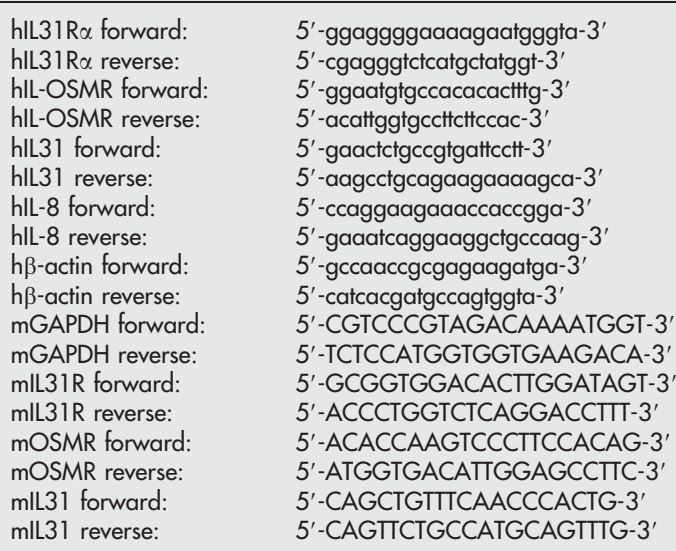

h: human; m: mouse. isolated using Trizol reagent (Gibco BRL/Life Technologies, Gaithersburg, MD, USA). For RT-PCR, RNA was treated with ribonuclease (RNase)-free deoxyribonuclease (DNA-free-Kit, Ambion) to remove potential genomic DNA contaminants. A volume of $2 \mu \mathrm{g}$ of total RNA was reverse transcribed using Qiagen Omniscript RT Kit. To control for genomic contamination, an identical parallel PCR reaction was performed containing starting material that had not been reverse transcribed. The following conditions were used for semi-quantitative PCRs: 40 cycles denaturing at $95^{\circ} \mathrm{C}$ for 30 seconds, annealing at $60^{\circ} \mathrm{C}$ for 30 seconds, extension at $72^{\circ} \mathrm{C}$ for 30 seconds. The primers (MWG Biotech, Ebersberg, Germany) for the PCR reactions are shown in table 1 . The PCR products were subcloned into pCR 2.1 vector (Invitrogen, Carlsbad, CA, USA) and sequenced.

\section{Quantitative PCR}

Real time PCR was performed with an ABI Prism 7700 Sequence Detection System (Applied Biosystems, Darmstadt, Germany) using the Quantitect SYBR Green PCR Kit from Qiagen (Hilden, Germany) following the manufacturer's guidelines. Oligonucleotide primers (table 1) were designed according to the published sequences. All mRNA expression levels were normalised to $\beta$-actin or GAPDH expression in the respective cDNA preparation.

\section{Signal transduction experiments, gel electrophoresis, and immunoblotting}

The signal transduction experiments were performed in overnight serum starved IEC lines as indicated. Cells were stimulated with $100 \mathrm{ng} / \mathrm{ml}$ IL31, unless indicated otherwise. This concentration was based on pilot experiments demonstrating a significantly higher effect of $100 \mathrm{ng} / \mathrm{ml}$ for the activation of certain kinases and cell migration than lower concentrations. Cells were solubilised in lysis buffer containing $1 \%$ Nonidet P40, 20 mM TRIS-HCl ( $\mathrm{pH} 7.4$ ), $150 \mathrm{mM} \mathrm{NaCl,} 2$ mM EDTA, $2 \mathrm{mM}$ EGTA, $10 \mu \mathrm{g} / \mathrm{ml}$ aprotinin, $2 \mathrm{mM}$ phenylmethylsulfonyl fluoride, $10 \mu \mathrm{g} / \mathrm{ml}$ leupeptin and phosphatase inhibitors (400 mM sodium orthovanadate, and $4 \mathrm{mM} \mathrm{NaF}$ ) and were passed six times through a 21 gauge needle. After 30 minutes on ice, lysates were cleared by centrifugation at $10000 \mathrm{~g}$ for 20 minutes. The protein concentration of each sample was quantified by the Bradford method. Immunoblotting was performed as previously described. ${ }^{13}$

\section{Immunofluorescence staining}

Immunofluorescence analysis of IL31R $\alpha$ expression in the IEC cell line HCT116 was performed as follows. Cells were fixed with $3.2 \%$ paraformaldehyde/PBS for 20 minutes and were then permeabilised with $0.5 \%$ Triton X-100/PBS for 5 minutes. Cells were blocked with $10 \%$ rabbit serum/PBS for 1 hour and then incubated with the primary antibody (anti-IL31R $\alpha, R \& D$ Systems) for 1 hour. After washing with PBS, cells were incubated with a FITC conjugated anti-goat secondary antibody (Sigma, Taufkirchen, Germany) and Hoechst 33342 (Sigma) for 1 hour. Cells were washed with PBS, mounted with Mowiol and were analysed under a fluorescence microscope. In negative controls, cells were stained omitting the primary antibody.

\section{Enzyme linked immunosorbent assay}

For the quantification of IL-8 release, BD OptEIA Human IL-8 ELISA Kit II (BD Biosciences, Bedford, MA, USA) was used according to the manufacturer's instructions.

\section{Cell proliferation and cell restitution assays}

HCT116 cells were seeded onto 96 well plates at densities of cells per well as indicated and were allowed to attach overnight. 
A

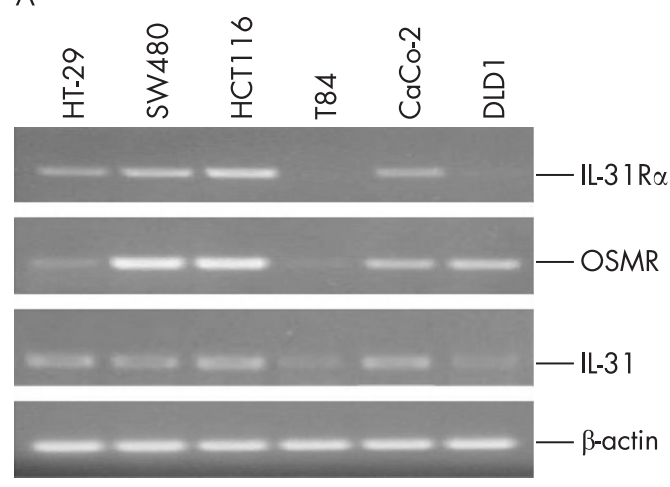

B

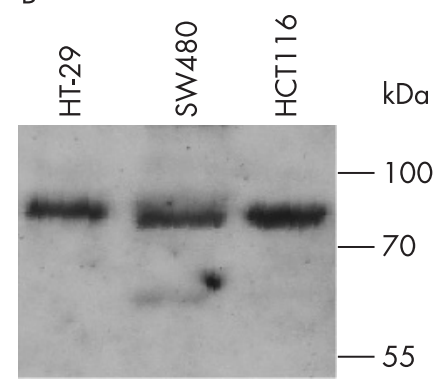

Figure 1 The IL31 receptor complex and IL31 are expressed in IEC. (A) mRNA expression of IL3IR $\alpha, O S M R$, and IL3I as analysed by RT-PCR analysis of mRNA derived from IEC lines as indicated. (B) Western blot analysis of total cell protein isolated from HT-29, SW480 and HCT1 16 cells demonstrates expression of the long, active isoform of IL3IR $\alpha$. An amount of $80 \mu \mathrm{g}$ protein was loaded per lane. (C) Immunofluorescence staining of HCT1 16 cells with an IL31R $\alpha$ specific antibody demonstrates cell surface expression of IL31R $\alpha$ (upper panels) while no specific staining was obtained with the secondary antibody only (lower panels).

\section{C}
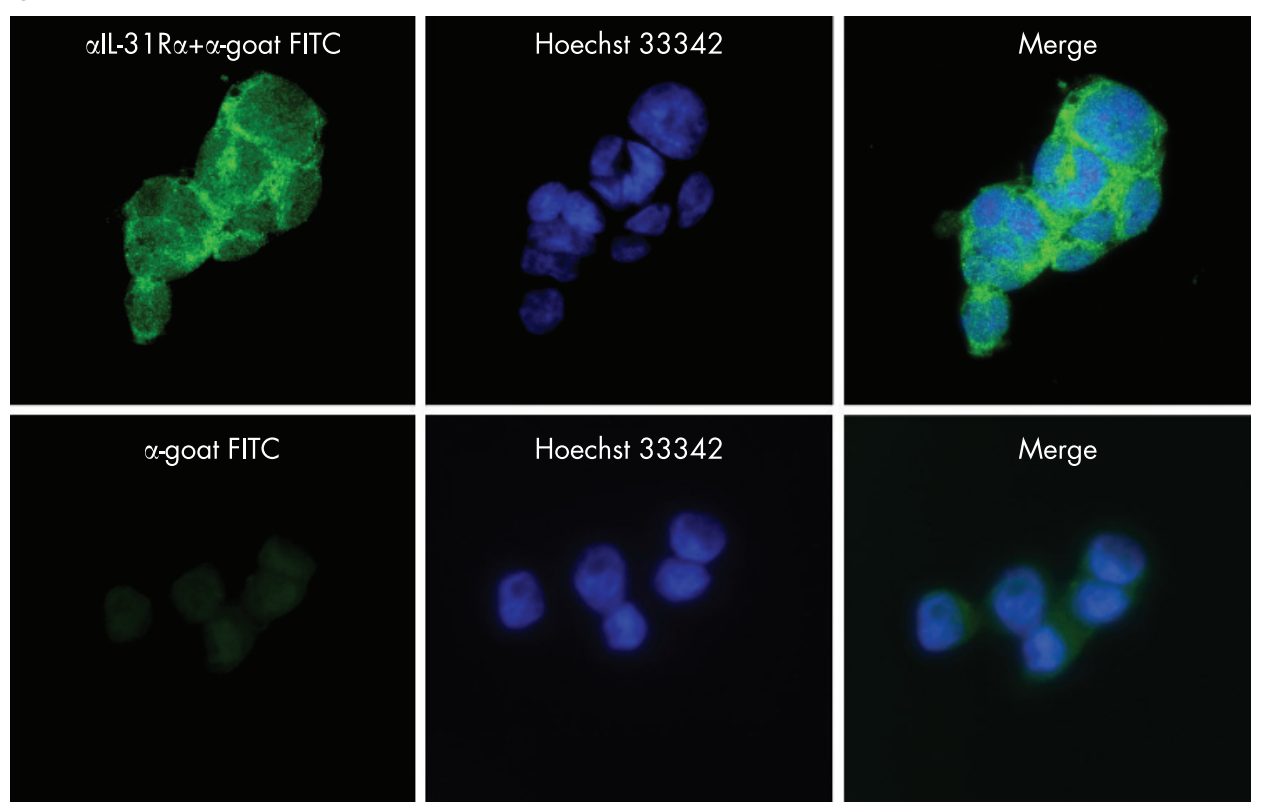

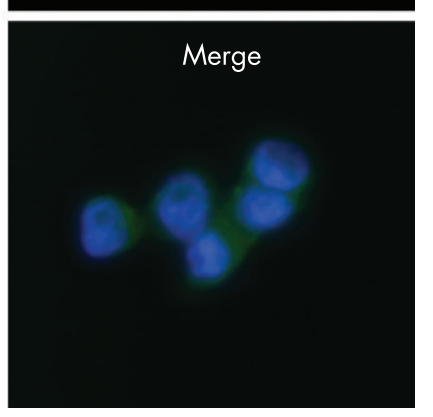

Cells were then stimulated with 10, 50, and $100 \mathrm{ng} / \mathrm{ml} \mathrm{IL31,} \mathrm{or}$ with cytokine-free medium (negative control) for 48 hours. The cell proliferation rate was determined by MTS assay on day 2 using the CellTiter 96 Aqueous Non-Radioactive Cell Proliferation Assay (Promega, Madison, WI, USA) according to the manufacturer's instructions.

Cell restitution assays were performed as wounding assays as previously described. ${ }^{14}$ Briefly, SW480 cells, which were the most suitable IEC line in pilot experiments, were grown in six well plates to complete confluence. Using a sterile razor blade, eight standardised wounds were created in each plate. Detached cells were removed by three washes with PBS, and the cell medium was changed from $10 \%$ FCS containing medium to $0.1 \%$ FCS containing medium. The cells were stimulated with IL31 (100 ng/ $\mathrm{ml}$ ) or PBS. The cells were washed with PBS after 24 hours and the number of migrated cells (over the wounding edge) was counted under a microscope. Five dishes were analysed for each group (IL31 stimulated and PBS stimulated), whereas for each dish eight separate fields were counted.

\section{Isolation of primary ileal epithelial cells from heterozygous TNF $\triangle$ ARE mice}

Heterozygous TNF $\triangle$ ARE mice (a generous gift from Dr G Kollias; Hellenic Pasteur Institute, Athens, Greece), which gradually develop chronic inflammation in the ileum from moderate to severe levels at 8 weeks and 18 weeks of age, ${ }^{15}$ and C57BL/6 wild type (WT) mice were killed at the age of
18 weeks. Primary IEC from the ileal and colonic epithelium of WT and TNF $\triangle \mathrm{ARE} / \mathrm{WT}$ mice were purified as previously described. ${ }^{16}$ Briefly, the intestinal tissue was cut into pieces and incubated at $37^{\circ} \mathrm{C}$ in DMEM containing 5\% FCS and $1 \mathrm{mM}$ dithiothreitol (DTT) for 30 minutes. The remaining tissue was incubated in $30 \mathrm{ml}$ PBS containing $1.5 \mathrm{mM}$ EDTA for additional 10 minutes. The supernatants were filtered, centrifuged for 5 minutes at $400 \mathrm{~g}$, and the cell pellet was resuspended in DMEM containing 5\% FCS. Finally, the primary IEC suspension was purified by centrifugation through a $25 \% / 40 \%$ discontinuous Percoll gradient at $600 \mathrm{~g}$ for 30 minutes. Cell purity was assessed by determining the absence of $\mathrm{CD} 3+\mathrm{T}$ cell contamination. Trypan blue exclusion confirmed the presence of at least $80 \%$ viable cells after the 2 hour isolation procedure. Primary IEC from the ileum were collected in sample buffer for subsequent RNA isolation.

\section{Murine cytomegalovirus infection in vivo}

C57BL/6 mice were infected intravenously with $1 \times 10^{6} \mathrm{pfu}$ murine cytomegalovirus (MCMV) of the Smith strain ${ }^{17}$ in PBS as previously described. ${ }^{18}$ Control mice received an injection of PBS only. After 45 hours, mice were killed by $\mathrm{CO}_{2}$ asphyxiation. Total RNA of the colon was isolated using Trizol reagent. The mice in vivo studies were approved by the animal care and use committee of the State of Bavaria (Regierung von Oberbayern) following the National Institutes of Health Guide for the Care and Use of Laboratory Animals. 
A



B



C

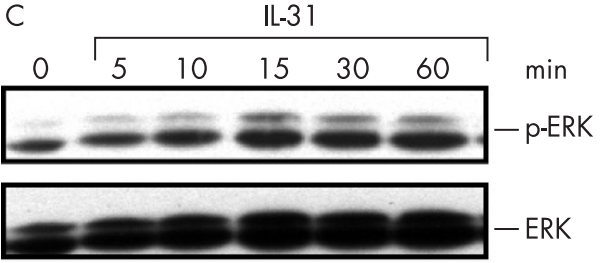

D

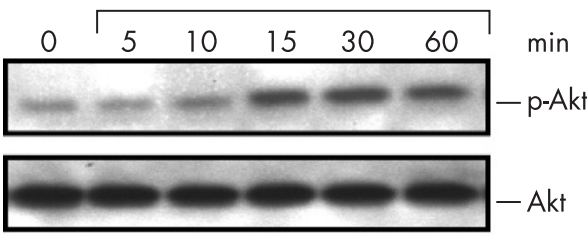

Figure 2 IL31 induces STAT1, STAT3, ERK-1/2, and Akt phosphorylation in IEC. Upon stimulation of HCT1 16 cells with IL31 $(100 \mathrm{ng} / \mathrm{ml})$, (A) STAT1 and (B) STAT3 proteins are phosphorylated. (C) Phospho-ERK-1/2 (p-ERK) activation after IL31 stimulation (100 ng/ml). (D) IL31 induces Akt phosphorylation. Similar results were obtained for SW480 cells (data not shown). One representative experiment $(n=3)$ is shown.

\section{Colonic biopsies}

Biopsies were taken from patients with CD and UC undergoing diagnostic colonoscopy. The study was approved by the ethics committee of the Medical Faculty of the University of Munich. All participating subjects gave written, informed consent before biopsy sampling. Four biopsies were collected from each patient: two from macroscopically non-inflamed sites and two from macroscopically inflamed mucosa. IL31, IL31R $\alpha$, OSMR, and IL8 mRNA levels were measured in each individual biopsy. For quantification, the average IL31, IL3 IR $\alpha$, or OSMR and IL8 mRNA expression of the two non-inflamed biopsies was compared to the average expression in the two inflamed biopsies. For calculation of the correlation coefficient, IL31 mRNA expression was correlated with the IL8 mRNA expression in the four individual biopsies for each patient.

\section{Statistical analysis}

Statistical analysis was performed using two tailed Student's $t$ test. p Values $<0.05$ were considered as significant.

\section{RESULTS}

The IL3 1 receptor complex is expressed in IEC lines Firstly, we determined if the IL31 receptor complex consisting of IL3IR $\alpha$ and OSMR is expressed in IEC using RT-PCR and CDNA derived from the human IEC lines HT-29, SW480, HCT116, T84, CaCo-2, and DLD-1. In addition, we examined IL31 expression in these cell lines. RT-PCR analysis demonstrated weak IL31 mRNA expression in all cell lines tested (fig lA). Using primers specific for the long isoform of this receptor, IL31R $\alpha$ expression was found in all IEC lines analysed with the exception of T84 and DLD-1 cells (fig 1A). OSMR was expressed in all cell lines although only at low levels in HT-29 and T84 cells (fig 1A). The long isoform of IL31R $\alpha$ was also expressed on protein level in IEC as demonstrated by western blot (fig 1B) and immunofluorescence staining (fig IC) with an IL31R $\alpha$ specific antibody. The strongest expression of both receptor subunits was demonstrated in SW480 and HCT116 cells (fig 1A and 1B), which we therefore used in the following signal transduction experiments.

\section{IL31 induces STAT1/3, ERK-1/2, and Akt phosphorylation}

After confirming IL31R $\alpha$ and OSMR expression in IEC, we next analysed if this complex is functional in IEC investigating various signalling pathways after stimulation of HCT116 and SW480 cells with recombinant IL31. Recent studies in other cell lines reported activation of STAT and MAP kinase signalling by IL31, ${ }^{14}$ which we therefore analysed in IEC. These experiments demonstrated moderate activation of both STATl (fig 2A) and STAT3 (fig 2B) in IEC after stimulation with IL31 ( $100 \mathrm{ng} / \mathrm{ml}$ ). Moreover, IL31 ( $100 \mathrm{ng} / \mathrm{ml}$ ) increased phosphorylation levels of ERK-1/2 (fig 2C) and Akt proteins (fig 2D).

\section{IL3 1 modulates IEC proliferation and migration}

The activation of ERK MAP kinases and Akt has been linked to increased cell proliferation, ${ }^{11}{ }^{12} 1920$ while a recent study demonstrated antiproliferative effects for IL31 in lung epithelial cells mediated via STAT3 activation. ${ }^{21}$ We therefore analysed the effect of IL31 on IEC proliferation using MTS assays and the IEC line HCT116. At cell densities of $<5000$ cells/well, IL31 strongly decreased cell proliferation $(p=0.001$ for 500 cells/well stimulated with $50 \mathrm{ng} / \mathrm{ml}$ or $100 \mathrm{ng} / \mathrm{ml} \mathrm{IL31}$, respectively, vs medium stimulated cells), while this effect was lost at higher cell densities and even an opposite, but much less pronounced proliferation-stimulating effect of IL31 (100 ng/ml) was observed with 10000 cells/well and 15000 cells/well compared to medium stimulated controls $(p=0.046$ and $p=0.013$, respectively; fig 3A).

Recently, we demonstrated that activation of Akt kinases and STAT proteins is also involved in IEC migration. ${ }^{11}$ Therefore, we next analysed the influence of IL31 on IEC migration. As demonstrated in figure 3B, $100 \mathrm{ng} / \mathrm{ml}$ IL3l increased the migration of HCT116 cells significantly $(p=0.005)$.

\section{IL3 1, IL3 I R $\alpha$, and OSMR mRNA expression is} upregulated in an in vitro model of cell differentiation Given the influence of cell density on the IL31 mediated effects on cell proliferation, we next analysed potential effects of IEC differentiation on IL31 and IL31 receptor expression in sodium butyrate treated IEC, which is an established in vitro model of cell differentiation. ${ }^{22}{ }^{23}$ We measured IL31, IL31R $\alpha$, and OSMR mRNA expression by quantitative PCR in HCTl16 cells after sodium butyrate treatment. There was an increased expression of all three genes that was most pronounced for IL31R $\alpha$ mRNA (270-fold upregulation after 48 hours, fig 3C), while IL31 and OSMR were upregulated up to 50-fold and 12-fold, respectively (fig 3C).

\section{IL3 1, IL3 IR $\alpha$, and OSMR mRNA expression is upregulated by proinflammatory cytokines and LPS} Given the high expression of IL31 in $\mathrm{T}$ cells, which are consistently found in various forms of intestinal inflammation, we next characterised expression and potential functions of IL3 I in inflammation. Firstly, we analysed IL31, IL31R $\alpha$, and OSMR mRNA expression in HCT116 cells after stimulation with 


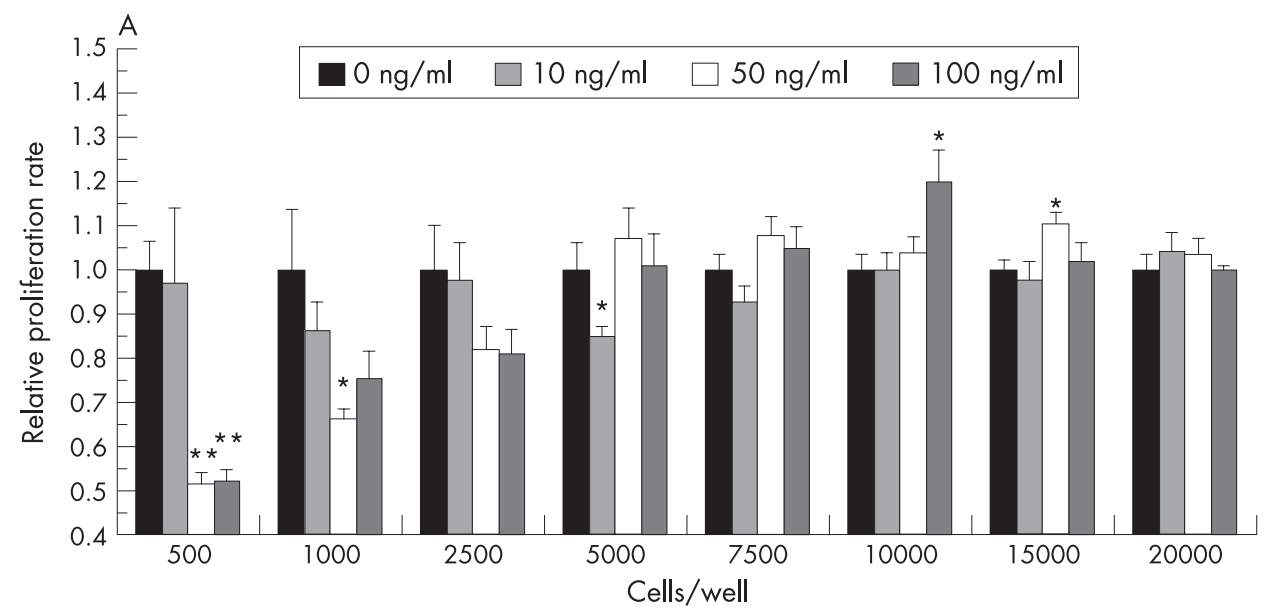

Figure 3 IL31 receptor expression is modulated by cell differentiation and influences IEC proliferation and migration. (A) IL31 influences cell proliferation depending on cell density as determined by MTS assay $(\mathrm{n}=6$ for each cytokine concentration and cell density; ${ }^{*} \mathrm{p}<0.05$, $\left.{ }^{* *} \mathrm{p}<0.005\right)$. Proliferation in unstimulated cells was arbitrarily set as 1.0 for each cell density. Data are presented as mean (SEM) (B) IL31 stimulation results in significantly increased cell migration in cell restitution assays $\left({ }^{*} p=0.005\right)$. Migration of unstimulated cells was defined as 1.0. (C) IL31, IL31R $\alpha$, and OSMR mRNA expression is strongly upregulated during cell differentiation induced by sodium butyrate.
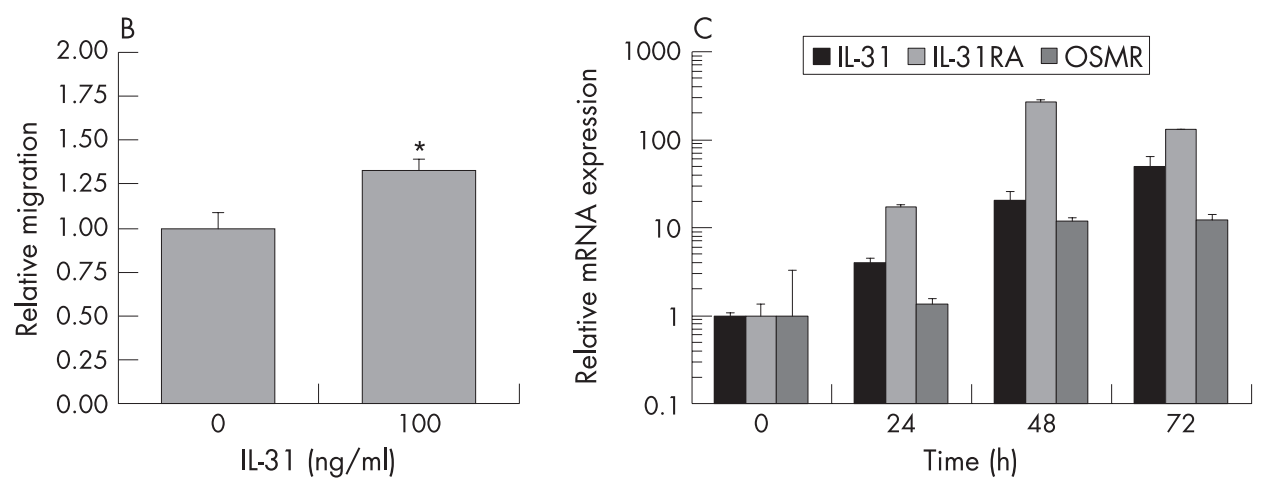

LPS and the proinflammatory cytokines TNF- $\alpha$, ILI $\beta$, and IFN$\gamma$. The mRNA expression of all three genes was upregulated 24 hours after stimulation with these proinflammatory cytokines (fig 4). The strongest effect on the transcriptional regulation of all three IL31 related genes was seen after stimulation with IFN- $\gamma$, which increased OSMR expression 5.4fold, IL3IR mRNA 3.5-fold, and IL31 mRNA 12.1-fold after 24 hours of stimulation.

\section{IL3 1 increases the expression of proinflammatory cytokines in IEC}

Based on the transcriptional upregulation of IL31 and its receptor complex by proinflammatory cytokines, we next analysed if IL31 itself regulates the expression of proinflammatory proteins. In this experiment, we studied in the IEC line HCT116 the effect of IL31 on protein expression of IL8, a prototypic inflammatory cytokine produced by IEC. ${ }^{24}$ As shown in figure 5, IL31 increased IL8 expression 3.1-fold after 24 hours of stimulation.

\section{IL3 I mRNA expression does not correlate with TNF- $\alpha$ mRNA expression in a Th 1 mediated model of murine ileitis}

Having shown that IL31, IL31R $\alpha$, and OSMR mRNAs are upregulated under inflammatory conditions in vitro, we next examined their expression in murine models of intestinal inflammation in vivo. Using heterozygous TNF $\triangle$ ARE mice as a model of chronic ileitis, ${ }^{15}$ we demonstrated no significant differences in IL31 mRNA expression in these mice compared to wild type mice of the same age and genetic background (fig 6A); however, there was a trend towards higher IL31 expression in colonic epithelial cells compared to ileal epithelial cells $(p=0.088)$. Similarly, OSMR expression was not significantly higher in TNF $\triangle$ ARE mice in comparison with wild type mice (fig 6B). In contrast, TNF $\triangle \mathrm{ARE}$ mice had a significantly higher TNF- $\alpha$ expression than wild type mice $(p<0.01$; fig $6 C)$.

\section{IL3 1 mRNA expression is not upregulated in MCMV infection in vivo}

In another set of experiments, we investigated colonic IL31, IL31R $\alpha$, and OSMR mRNA expression in viral infection using the murine in vivo model of MCMV infection. C57BL/6 mice were infected with $10^{6}$ pfu MCMV of the Smith strain ${ }^{17}$ as previously described..$^{18}$ However, 45 hours after infection, IL31 and IL31R $\alpha$ mRNA levels were not significantly different compared to non-infected mice $(p=0.73$; fig 6D) while there was a trend towards a higher OSMR expression in infected mice $(\mathrm{p}=0.06$; fig 6D). In contrast, in TNF $\triangle \mathrm{ARE}$ mice and corresponding wild type mice, IL31R $\alpha$ mRNA levels were lower and just above the detection threshold in both mice strains without significant differences between the two strains (data not shown). However, in comparison with the cDNA isolation of the MCMV infected mice (fig 6D), the isolation procedure of ileal and colonic epithelial cells of TNF $\triangle$ ARE mice yielded much lower RNA and cDNA amounts, respectively. Using similar reaction volumes, there was GAPDH detection already after 18 PCR cycles in the MCMV infected mice, while GAPDH was detected in TNF $\triangle$ ARE mice only after 25 PCR cycles, explaining the different levels of IL31R $\alpha$ expression in two mouse experiments.

\section{IL3 1 mRNA expression is increased in the inflamed colonic mucosa of patients with inflammatory bowel disease}

We next analysed IL31 and its receptor expression in human intestinal inflammation in vivo using real time PCR. In this analysis, we compared IL31, IL31R $\alpha$, and OSMR mRNA expression levels in a total of 88 biopsy samples taken from 22 different patients with inflammatory bowel disease (IBD) 

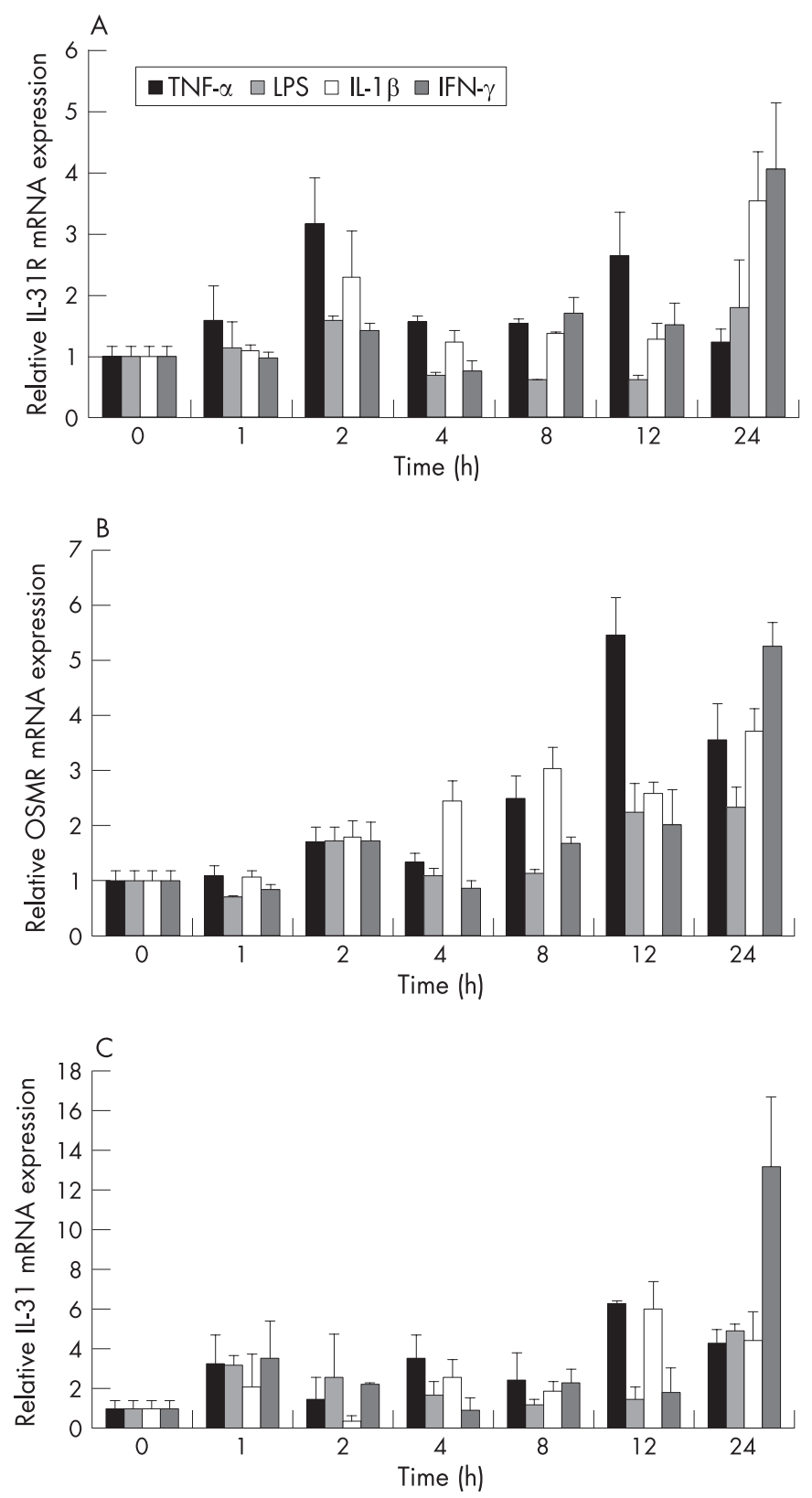

Figure 4 IL3IR $\alpha$, OSMR, and IL31 mRNA expression are upregulated by proinflammatory stimuli. (A) Regulation of IL31R $\alpha$, (B) OSMR, and (C) IL31 by LPS $(1 \mu \mathrm{g} / \mathrm{ml})$ and the proinflammatory cytokines TNF- $\alpha(50 \mathrm{ng} / \mathrm{ml})$, IL1 $\beta(10 \mathrm{ng} / \mathrm{ml})$, and IFN- $\gamma(1000 \mathrm{U} / \mathrm{ml})$. HCT1 16 cells were stimulated for time intervals as indicated and mRNA expression was measured by quantitative PCR and normalised to $\beta$-actin expression.

including 12 patients with CD and 10 patients with UC. The biopsies were sampled from sites with endoscopically (macroscopic) inflamed colonic mucosa and compared to those of endoscopically non-inflamed colonic mucosa taken from the same 22 patients. We used the IL8 mRNA expression in these biopsies as a control marker of inflammation. IL8 mRNA expression was significantly increased $(\mathrm{p}<0.05)$ in the inflamed biopsy samples in CD (fig 7A). Moreover, in CD IL31 mRNA expression levels were higher in inflamed colonic biopsy samples than in non-inflamed colonic lesions (mean increase 2.4-fold; fig 7A) and correlated with IL8 mRNA expression in these patients $(\mathrm{r}=0.564$; fig $7 \mathrm{C})$. There was also a twofold higher IL3IR $\alpha$ and OSMR mRNA expression in inflamed biopsies compared to uninflamed tissue (fig 7A). Similarly, in eight of the 10 patients with UC the IL31 mRNA

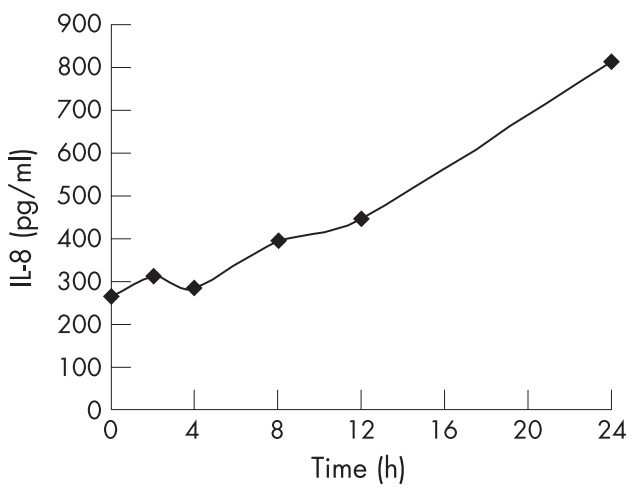

Figure 5 IL31 increases IL8 expression in IEC. IL8 protein levels increase and reach a maximum after 24 hours of IL31 stimulation. IL8 protein concentration was determined by ELISA.

expression was increased in the inflamed lesions (mean increase 2.6-fold) and correlated with the IL8 mRNA expression in these lesions $(r=0.650$; fig $7 \mathrm{~B}, \mathrm{D})$. In addition, IL3 $\mathrm{IR} \alpha$ and OSMR mRNA expression was slightly increased in the inflamed tissue of the UC patients (1.5-fold and 1.7-fold, fig 7B).

\section{DISCUSSION}

Crohn's disease and ulcerative colitis, the two main forms of inflammatory bowel disease, are defined as idiopathic, chronic relapsing, inflammatory conditions that are immunologically mediated..$^{25}$ Despite intensive research their aetiology is only incompletely understood..$^{25}{ }^{26}$ However, several cytokines such as TNF- $\alpha$, IL1 $\beta$, and IL23 play a key part in the aggravation of intestinal inflammation. ${ }^{25-30}$ Given its important role in inflammatory disorders of the skin, ${ }^{16-10}$ we therefore analysed in this study the novel cytokine IL31 focusing on expression, signalling, and its role in intestinal inflammation.

While activated T cells are the main source of IL31, ${ }^{1}$ we here demonstrate that IEC lines express the functional IL31 receptor complex consisting of IL3IR $\alpha$ and OSMR, which is consistent with the expression pattern shown for other epithelial cell types such as keratinocytes ${ }^{1}$ and lung epithelial cells. ${ }^{4}$ After stimulation with IL31, ERK, Akt, and STAT proteins are activated in IEC. Similar to our findings, activation of STATI/3 and PI3 kinase signalling has been observed in glioblastoma and melanoma tumour cells, ${ }^{2}$ and in lung epithelial cells ${ }^{4}$ following IL31 stimulation. However, in agreement with a recent study in lung epithelial cells, in which strong activation was only obtained by IL31 receptor overexpression, ${ }^{4}$ the level of STAT activation in non-transfected IEC was only moderate.

Recently, we demonstrated that activation of Akt and STAT proteins mediates IEC proliferation and migration. ${ }^{11} 121920$ Similarly, our experiments demonstrated that IL31 receptor activation increases IEC migration. Interestingly, IL31 is located on chromosome 12q24 in close proximity to chemokine-like factor 1 , which has been very recently shown to be a functional ligand for CCR4. ${ }^{31}$ Given the results of our experiments, IL31 also has chemotactic properties influencing the migration of IEC. Moreover, an influence on cell migration has been demonstrated for the IL31 related cytokine IL- $6^{32}{ }^{33}$ and several other IL6-like cytokines such as LIF. ${ }^{34}$ In addition, it has been shown that LIF may upregulate the gene expression of chemokines such as MCP-1. ${ }^{34}$ Similarly, we here show that IL31 increases the expression of the proinflammatory chemokine IL8. Particularly the activation of STAT3 is a common signalling pathway mediated by these migration stimulating cytokines. ${ }^{35}$ For example, we recently demonstrated that cell migration mediated by the IL-10-like cytokine IL22 is dependent on STAT3 activation. ${ }^{11}$ A very recent study 



Figure 6 IL31 mRNA expression is not increased in murine models of Th 1 mediated intestinal inflammation. (A) IL31 expression was similar in ileal and colonic epithelial cells of TNF $\triangle$ ARE mice $(n=9)$ compared to C57BL/ 6 wild type mice $(n=9)$. Ileal mRNA expression in wild type mice was arbitrarily set to 1.0 and all other expression levels were calculated proportionally as fold increase. Data are presented as mean (SEM). (B) OSMR mRNA expression was not significantly different in TNF $\triangle A R E$ mice compared to wild type mice $(p=0.26)$. (C) TNF- $\alpha$ mRNA expression was significantly higher in TNF $\triangle A R E$ mice compared to wild type mice $(p<0.01)$. (D) IL31, IL31R $\alpha$, and OSMR mRNA expression were not significantly increased in C57BL/6 mice infected with murine cytomegalovirus $(n=10)$ compared to uninfected C57BL/6 mice $(n=4)$. established that OSMR mediated signal transduction is negatively regulated by SOCS3 through a receptor tyrosine independent mechanism. ${ }^{36}$ Consistently, we demonstrated that SOCS3 can also inhibit signalling of other STAT activating cytokines. ${ }^{37-42}$

IL31R $\alpha$ belongs to the IL6 cytokine receptor (IL6R) family. IL6Rs have divergent effects on cell proliferation in different epithelial cell types. While suppression of proliferation has been described for IL6R mediated signalling in some epithelial cells, ${ }^{43}$ it enhances cell growth in other epithelial cell lines. ${ }^{44}{ }^{45}$ Here, we demonstrate that IL31 at high doses and low cell density significantly decreases cell proliferation in the colorectal cancer cell line HCT116. Similarly, a very recent study demonstrated that IL3 I was highly effective in suppressing proliferation by altering expression of cell cycle proteins including upregulation of p27Kipl and downregulation of cyclinB1, cdc2, cdk6, mcm4, and $\mathrm{Rb}$ in lung epithelial cells. ${ }^{4}$ Here, we demonstrate that the antiproliferative effect of IL31 in HCT116 cells was lost in dense cell cultures and under certain conditions even reversed into a proliferation stimulating effect. A similar bimodal effect on cell
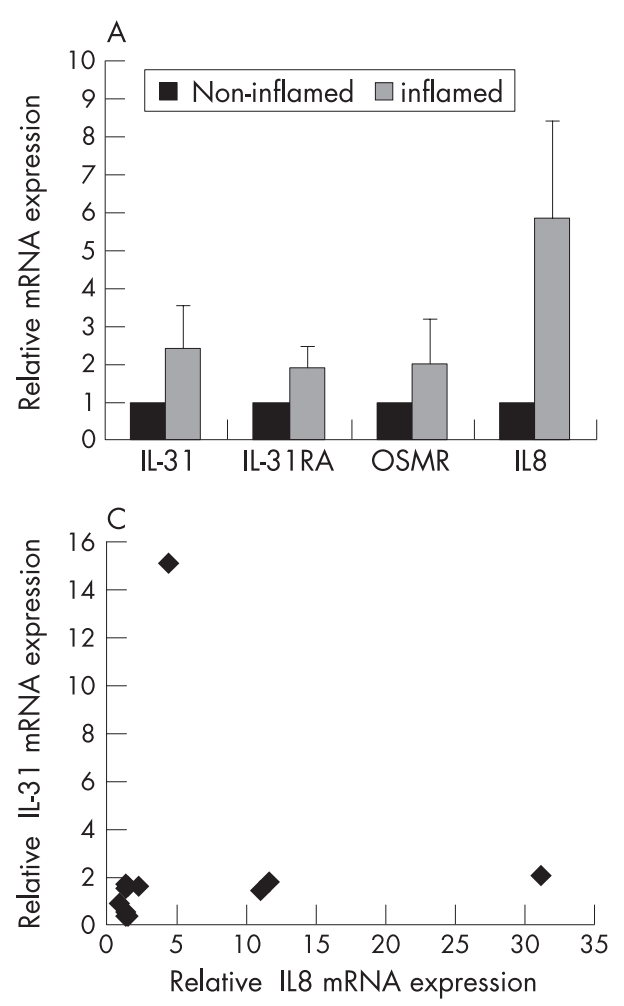
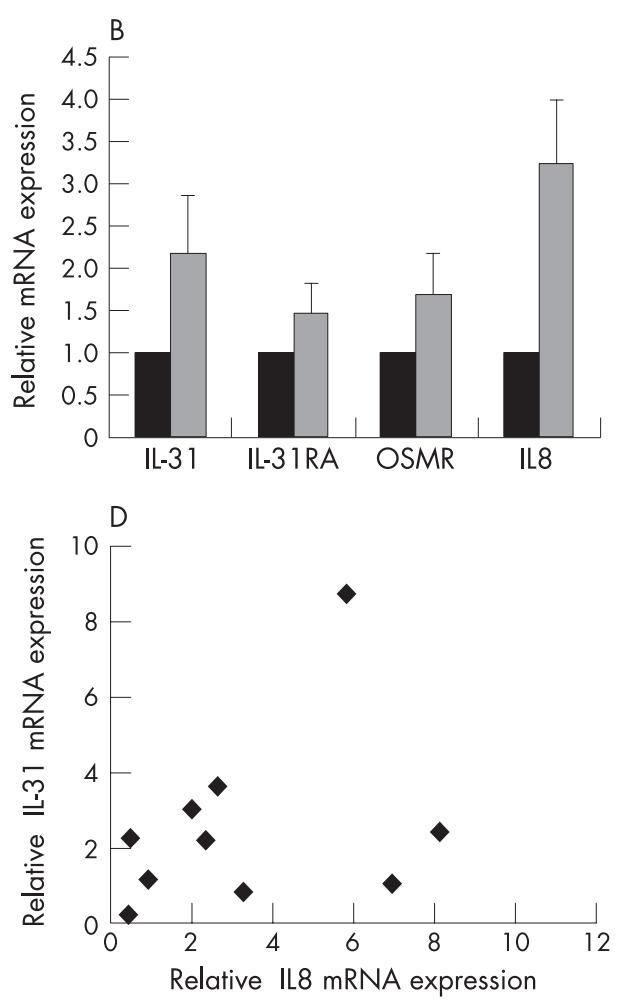

Figure 7 Colonic IL31 mRNA expression is increased in human inflammatory bowel disease. (A, C) IL31 expression is increased 2.4-fold in inflamed colonic biopsies from patients with Crohn's disease compared to uninflamed biopsies while IL3IR $\alpha$ and OSMR expression were increased twofold. IL31 mRNA expression correlated with the IL8 expression $(r=0.56)$, which was measured as a marker for inflammation. (B, D) In patients with ulcerative colitis, a 2.6 -fold increased IL31 mRNA expression was observed in inflamed biopsies in comparison with uninflamed tissue with a high correlation to IL8 mRNA levels $(r=0.65)$. IL31R $\alpha$ und OSMR were increased 1.5-fold and 1.7-fold, respectively. 
proliferation dependent on cell density has also been described for other cytokines such as TGF- $\beta .^{46}$ However, a number of studies demonstrated that unstimulated cells in dense cultures undergoing contact inhibition are characterised by similar alterations of the expression of cell cycle proteins as caused by IL31 stimulation ${ }^{4}$ including downregulation of cyclins $^{47}$ and upregulation of $\mathrm{p} 27 \mathrm{Kipl}{ }^{48}$ which explains the relative loss of the antiproliferative effect of IL31 (in comparison with unstimulated cells) in dense cultures considering the overall low cell proliferation in (unstimulated) dense cultures. Similar to IL31, other proliferation inhibiting agents such as bone morphogenetic protein (BMP-7) $)^{49}$ and the MEK inhibitors PD98059 and U0126 $6^{47}$ have much higher effects on cell proliferation inhibition at low cell density compared to dense cultures. In contrast, cell proliferation stimulating cytokines such as hepatocyte growth factor (HGF) lose their proliferation stimulating properties in cell cultures with low cell density. ${ }^{47}$ It has been demonstrated that a single STAT3 recruitment site (Tyr-721) in the cytoplasmic domain of IL31R $\alpha$ exerts a dominant function in the entire receptor complex and is critical for gene induction and growth inhibition. ${ }^{4}$ Similarly, we demonstrated upregulation of STAT3 following IL31 stimulation in our study. However, in the initial study, ${ }^{4}$ an IL31R $\alpha$ overexpressing cell line was used explaining the stronger antiproliferative effects of IL31 observed in that study compared to our experiments. In addition, it has been demonstrated that high cell density itself is associated with ligand independent STAT3 activation mediating growth inhibition, ${ }^{50}$ which further explains the loss of the antiproliferative properties of IL31 in dense cell cultures. Moreover, we demonstrate that IL31 and IL31 receptor mRNA expression are cell differentiation dependent, which further supports the strong influence of cell density on the biological functions of IL31.

In this study, we demonstrate that proinflammatory cytokines increase the expression of IL31 and its receptor complex. The strongest upregulation of IL31, IL31R $\alpha$, and OSMR gene expression was seen after stimulation with IFN- $\gamma$. Similarly, in monocytes, gene upregulation of IL31R $\alpha$ was stronger following IFN- $\gamma$ compared to LPS stimulation. ${ }^{1}$ Given our quantitative PCR results of primary murine IEC, which demonstrated in comparison to colorectal cancer derived IEC only weak IL31R $\alpha$ mRNA expression, it is therefore likely that proinflammatory cytokine activated monocytes are a major IL31R $\alpha$ expressing cell type and target of IL31 in IBD.

Depending on the $\mathrm{T}$ cell source of cytokine production, cytokines have been differentiated in Thl and Th2 cytokines. It has been proposed that CD represents a Thl and Thl7 mediated intestinal inflammation, while ulcerative colitis resembles more a Th2 mediated colitis. ${ }^{3045}$ IL31 is mainly produced by activated Th2 cells ${ }^{1}$ and a very recent study implicates IL3IR signalling as a novel negative regulatory pathway that specifically limits Th2 mediated inflammation ${ }^{52}$ while development of intestinal pathology in the TNF $\triangle$ ARE mouse model depends on Thl-like cytokines such as IL12 and IFN- $\gamma .^{53}$ In contrast, the generation of $\mathrm{CD} 4(+) \mathrm{T}$ cell mediated Thl responses were normal in $\operatorname{IL} 31 \mathrm{R} \alpha(-/-)$ mice. ${ }^{52}$ Consistently, we demonstrated no increased IL31 mRNA expression in TNF $\triangle$ ARE mice, a Thl mediated murine model of ileitis. ${ }^{15} 53$ In these mice, baseline colonic IL31 expression was higher than ileal IL3 1 expression. This is in agreement with the first report on IL31 expression in normal tissues demonstrating a higher IL31 expression in the colon than in the small intestine. ${ }^{1}$ However, in comparison with uninflamed colonic tissue, the IL31 mRNA expression was upregulated in inflamed colonic tissue in both forms of IBD suggesting that in humans IL31 mediated effects are not completely restricted to Th2 mediated types of inflammation. Moreover, the IL31 mRNA expression correlated with IL8 mRNA expression in both forms of intestinal inflammation.

While this is the first report on IL31 in intestinal inflammation, the role of IL31 in skin inflammation, particularly in atopic dermatitis is well established. ${ }^{17-10}$ Interestingly, there is an association between intestinal inflammation and atopic dermatitis. ${ }^{545}$ Moreover, in patients with atopic dermatitis, disturbances in intestinal permeability compared to normal controls have been reported..$^{56}{ }^{57}$ Therefore, IL31 producing $\mathrm{T}$ cells may mediate skin reactions as observed in many IBD patients but this and the role of IL3I on intestinal permeability need further investigation. Given its effect demonstrated here on IEC proliferation and migration, it is likely that this novel cytokine system modulates IEC barrier function. However, while most colorectal cancer derived IEC expressed considerable levels of IL3IR $\alpha$, its mRNA expression levels in unstimulated murine primary IEC were low suggesting that cancerogenesis might be another process in which this novel cytokine is involved. This is supported by IL31R $\alpha$ expression in other malignancies such as glioblastoma and melanoma, ${ }^{2}$ which also secrete a soluble form of OSMR which may bind IL31 when combined with soluble IL31R $\alpha,{ }^{58}$ but further studies are required to elucidate potential functions of IL31 in cancerogenesis.

In summary, we demonstrated that IEC express the functional IL31 receptor complex. Binding of IL31 to its surface receptor complex in IEC leads to phosphorylation of STATI/3, Akt, and ERK MAP kinases. IL31 inhibited cell proliferation in low density cell cultures and increased IEC migration. Moreover, the mRNA expression of IL31 was upregulated in inflamed colonic lesions of IBD patients but not in a Thl mediated model of murine ileitis. Taken together, our data indicate a role for this cytokine in promoting proinflammatory gene expression and modulating IEC barrier function suggesting a role in the pathogenesis of IBD.

\section{ACKNOWLEDGEMENTS}

We thank G Spöttl and K Zitzmann (University of Munich) for excellent technical support. This work contains parts of the unpublished doctoral thesis of J Dambacher at Ludwig-Maximilians-University Munich, Germany. This study was supported by grants of the Deutsche Forschungsgemeinschaft (BR1912/5-1), Else-Kröner-FreseniusStiftung (P60/05//EKMS 05/62), and Friedrich-Baur-Stiftung. JD was supported by a grant from the University of Munich (Promotionsstipendium). JS was supported by a grant from the University of Munich (Habilitationsstipendium).

\section{Authors' affiliations \\ Julia Dambacher, Florian Beigel, Julia Seiderer, Burkhard Göke, Christoph J Auernhammer, Stephan Brand, Department of Medicine II, University Hospital Munich-Grosshadern, University of Munich, Germany Dirk Haller, Nutrition and Food Research Center, Experimental Nutritional Medicine, Technical University Munich-Weihenstephan, Germany \\ Competing interests: None. \\ REFERENCES \\ 1 Dillon SR, Sprecher C, Hammond A, et al. Interleukin 31, a cytokine produced by activated T cells, induces dermatitis in mice. Nat Immunol 2004;5:752-60. \\ 2 Diveu C, Lak-Hal AH, Froger J, et al. Predominant expression of the long isoform of GP130-like (GPL) receptor is required for interleukin-31 signaling. Eur Cytokine Netw 2004;15:291-302. \\ 3 Dreuw A, Radtke S, Pflanz S, et al. Characterization of the signaling capacities of the novel gp130-like cytokine receptor. J Biol Chem 2004;279:36112-20. \\ 4 Chattopadhyay S, Tracy E, Liang $P$, et al. Interleukin-31 and oncostatin-M mediate distinct signaling reactions and response patterns in lung epithelial cells. J Biol Chem 2007;282:3014-26. \\ 5 Kuropatwinski KK, De Imus C, Gearing D, et al. Influence of subunit combinations on signaling by receptors for oncostatin $M$, leukemia inhibitory factor, and interleukin-6. J Biol Chem 1997;272:15135-44.}


6 Neis MM, Peters B, Dreuw A, et al. Enhanced expression levels of IL31 correlate with IL-4 and IL-13 in atopic and allergic contact dermatitis. J Allergy Clin Immunol 2006:118:930-7.

7 Sonkoly E, Muller A, Laverma Al, et al. IL31: a new link between T cells and pruritus in atopic skin inflammation. J Allergy Clin Immunol 2006;117:41 1-7.

8 Bilsborough J, Leung DY, Maurer M, et al. IL31 is associated with cutaneous lymphocyte antigen-positive skin homing $T$ cells in patients with atopic dermatitis. J Allergy Clin Immunol 2006; 1 17:418-25.

9 Takaoka A, Arai I, Sugimoto M, et al. Expression of IL31 gene transcripts in NC/ Nga mice with atopic dermatitis. Eur J Pharmacol 2005;516:180-1.

10 Takaoka A, Arai I, Sugimoto M, et al. Involvement of IL31 on scratching behavior in NC/Nga mice with atopic-like dermatitis. Exp Dermatol 2006;15:161-7.

11 Brand S, Beigel F, Olszak T, Zitzmann K, et al. IL-22 is increased in active Crohn's disease and promotes proinflammatory gene expression and intestinal epithelial cell migration. Am J Physiol Gastrointest Liver Physiol 2006;290:G827-38.

12 Brand S, Sakaguchi T, Gu X, et al. Fractalkine-mediated signals regulate cellsurvival and immune-modulatory responses in intestinal epithelial cells. Gastroenterology 2002;122:166-77.

13 Muehlhoefer A Saubermann $\sqcup, G u X$ et al. Fractalkine is an epithelial and endothelial cell-derived chemoattractant for intraepithelial lymphocytes in the small intestinal mucosa. J Immunol 2000;164:3368-76.

14 Dignass AU, Podolsky DK. Cytokine modulation of intestinal epithelial cell restitution: central role of transforming growth factor beta. Gastroenterology 1993; 105:1323-32.

15 Kontoyiannis D, Pasparakis M, Pizarro TT, et al. Impaired on/off regulation of TNF biosynthesis in mice lacking TNF AU-rich elements: implications for joint and gut-associated immunopathologies. Immunity 1999;10:387-98.

16 Ruiz PA, Shkoda A, Kim SC, et al. IL-10 gene-deficient mice lack TGF-beta/ Smad signaling and fail to inhibit proinflammatory gene expression in intestinal epithelial cells after the colonization with colitogenic Enterococcus faecalis. J Immunol 2005; 174:2990-9.

17 Rawlinson WD, Farrell HE, Barrell BG. Analysis of the complete DNA sequence of murine cytomegalovirus. J Virol 1996;70:8833-49.

18 Brand S, Beigel F, Olszak T, et al. IL-28A and IL-29 mediate antiproliferative and antiviral signals in intestinal epithelial cells and murine CMV infection increases colonic IL-28A expression. Am J Physiol Gastrointest Liver Physiol 2005;289:G960-8.

19 Brand S, Dambacher J, Beigel F, et al. CXCR4 and CXCL12 are inversely expressed in colorectal cancer cells and modulate cancer cell migration, invasion and MMP-9 activation. Exp Cell Res 2005:310:117-30.

20 Brand S, Olszak T, Beigel F, et al. Cell differentiation dependent expressed CCR6 mediates ERK-1/2, SAPK/JNK, and Akt signaling resulting in proliferation and migration of colorectal cancer cells. J Cell Biochem 2006;97:709-23.

21 Chattopadhyay S, Tracy E, Liang $P$, et al. Interleukin-31 and oncostatin-M mediate distinct signaling reactions and response patterns in lung epithelial cells. J Biol Chem 2007;282:3014-26.

22 Chung YS, Song IS, Erickson RH, et al. Effect of growth and sodium butyrate on brush border membrane-associated hydrolases in human colorectal cancer cell lines. Cancer Res 1985;45:2976-82.

23 Jordan NJ, Kolios G, Abbot SE, et al. Expression of functional CXCR4 chemokine receptors on human colonic epithelial cells. J Clin Invest 1999;104:1061-9.

24 Eckmann L, Jung HC, Schurer-Maly C, et al. Differential cytokine expression by human intestinal epithelial cell lines: regulated expression of interleukin 8. Gastroenterology 1993;105:1689-97.

25 Sartor RB. Mechanisms of disease: pathogenesis of Crohn's disease and ulcerative colitis. Nat Clin Pract Gastroenterol Hepatol 2006;3:390-407

26 Podolsky DK. Inflammatory bowel disease. N Engl J Med 2002;347:417-29.

27 MacDonald $T$, Hutchings $P$, Choy MY, et al. Tumour necrosis factor-alpha and interferon-gamma production measured at the single cell level in normal and inflamed human intestine. Clin Exp Immunol 1990;81:301-5.

28 Murch SH, Lamkin VA, Savage MO, et al. Serum concentrations of tumour necrosis factor alpha in childhood chronic inflammatory bowel disease. Gut $1991 ; 32: 913-7$.

29 Satsangi J, Wolstencroft RA, Cason J, et al. Interleukin 1 in Crohn's disease. Clin Exp Immunol 1987;67:594-605.

30 Neurath MF. IL-23: a master regulator in Crohn disease. Nat Med 2007;13:26-8.

31 Wang $Y$, Zhang $Y$, Yang $X$, et al. Chemokine-like factor 1 is a functional ligand for CC chemokine receptor 4 (CCR4). Life Sci 2006;78:614-21.

32 Weissenbach M, Clahsen T, Weber C, et al. Interleukin- 6 is a direct mediator of T cell migration. Eur J Immunol 2004;34:2895-906.

33 Yao JS, Zhai W, Young WL, et al. Interleukin-6 triggers human cerebral endothelial cells proliferation and migration: the role for KDR and MMP-9. Biochem Biophys Res Commun 2006;342:1396-404.

34 Tofaris GK, Patterson PH, Jessen KR, et al. Denervated Schwann cells attract macrophages by secretion of leukemia inhibitory factor (LIF) and monocyte chemoattractant protein- 1 in a process regulated by interleukin- 6 and LIF. J Neurosci 2002;22:6696-703.

35 McLoughlin RM, Jenkins BJ, Grail D, et al. IL-6 trans-signaling via STAT3 directs T cell infiltration in acute inflammation. Proc Natl Acad Sci USA 2005; 102:9589-94.

36 Stross C, Radtke S, Clahsen $T$, et al. Oncostatin M receptor-mediated signal transduction is negatively regulated by SOCS3 through a receptor tyrosineindependent mechanism. J Biol Chem 2006;281:8458-68.

37 Brand S, Zitzmann K, Dambacher J, et al. SOCS-1 inhibits expression of the antiviral proteins $2^{\prime}, 5^{\prime}$-OAS and $M \times A$ induced by the novel interferon-lambdas IL-28A and IL-29. Biochem Biophys Res Commun 2005;331:543-8.

38 Auernhammer CJ, Chesnokova V, Bousquet C, et al. Pituitary corticotroph SOCS-3: novel intracellular regulation of leukemia-inhibitory factor-mediated proopiomelanocortin gene expression and adrenocorticotropin secretion. Mol Endocrinol 1998;12:954-61.

39 Auernhammer CJ, Isele NB, Kopp FB, et al. Novel neurotrophin-1/B cellstimulating factor-3 (cardiotrophin-like cytokine) stimulates corticotroph function via a signal transducer and activator of transcription-dependent mechanism negatively regulated by suppressor of cytokine signaling-3. Endocrinology 2003;144:1202-10

40 Vlotides G, Sorensen AS, Kopp F, et al. SOCS-1 and SOCS-3 inhibit IFN-alphainduced expression of the antiviral proteins 2,5-OAS and MxA. Biochem Biophys Res Commun 2004;320:1007-14.

41 Zitzmann K, Brand S, Baehs S, et al. Novel interferon-lambdas induce antiproliferative effects in neuroendocrine tumor cells. Biochem Biophys Res Commun 2006;344:1334-41

42 Brand S, Dambacher J, Beigel F, et al. IL-22 mediated liver cell regeneration is abrogated by SOCS-1/3 overexpression in vitro. Am J Physiol Gastrointest Liver Physiol 2007;292:G1019-28.

43 Grant SL, Douglas AM, Goss GA, et al. Oncostatin M and leukemia inhibitory factor regulate the growth of normal human breast epithelial cells. Growth Factors 2001; 19:153-62.

$44 \mathrm{Yu} \mathrm{H}$, Jove R. The STATs of cancer-new molecular targets come of age. Nat Rev Cancer 2004:4:97-105.

45 Garcia R, Bowman TL, Niu G, et al. Constitutive activation of Stat3 by the Src and JAK tyrosine kinases participates in growth regulation of human breast carcinoma cells. Oncogene $2001 ; 20: 2499-513$.

46 Boumediene K, Takigawa M, Pujol JP. Cell density-dependent proliferative effects of transforming growth factor (TGF)-beta 1, beta 2, and beta 3 in human chondrosarcoma cells HCS-2/8 are associated with changes in the expression of TGF-beta receptor type I. Cancer Invest 2001;19:475-86.

47 Li S, Gerrard ER Jr, Balkovetz DF. Evidence for ERK1/2 phosphorylation controlling contact inhibition of proliferation in Madin-Darby canine kidney epithelial cells. Am J Physiol Cell Physiol 2004;287:C432-9.

48 Yanagisawa K, Kosaka A, Iwahana $\mathrm{H}$, et al. Opposite regulation of the expression of cyclin-dependent kinase inhibitors during contact inhibition. J Biochem (Tokyo) 1999;125:36-40.

49 Franzen A, Heldin NE. BMP-7-induced cell cycle arrest of anaplastic thyroid carcinoma cells via p21(CIP1) and p27(KIP1). Biochem Biophys Res Commun $2001 ; 285: 773-81$.

50 Steinman RA, Wentzel A, Lu Y, et al. Activation of Stat3 by cell confluence reveals negative regulation of Stat3 by cdk2. Oncogene 2003;22:3608-15.

51 Bouma G, Strober W. The immunological and genetic basis of inflammatory bowel disease. Nat Rev Immunol 2003;3:521-33.

52 Perrigoue JG, Li J, Zaph C, et al. IL31-IL31R interactions negatively regulate type 2 inflammation in the lung. J Exp Med 2007;204:481-7.

53 Kontoyiannis D, Boulougouris G, Manoloukos M, et al. Genetic dissection of the cellular pathways and signaling mechanisms in modeled tumor necrosis factorinduced Crohn's-like inflammatory bowel disease. J Exp Med 2002;196:1563-74.

54 Niwa $Y$, Sumi H, Akamatsu $H$. An association between ulcerative colitis and atopic dermatitis, diseases of impaired superficial barriers. J Invest Dermatol 2004; 123:999-1000.

55 Majamaa H, Miettinen A, Laine S, et al. Intestinal inflammation in children with atopic eczema: faecal eosinophil cationic protein and tumour necrosis factoralpha as non-invasive indicators of food allergy. Clin Exp Allergy 1996;26:181-7.

56 Jackson PG, Lessof MH, Baker RW, et al. Intestinal permeability in patients with eczema and food allergy. Lancet 1981;1:1285-6.

57 Dupont C, Barau E, Molkhou P, et al. Food-induced alterations of intestinal permeability in children with cow's milk-sensitive enteropathy and atopic dermatitis. J Pediatr Gastroenterol Nutr 1989;8:459-65.

58 Diveu C, Venereau E, Froger J, et al. Molecular and functional characterization of a soluble form of oncostatin $\mathrm{M}$ /interleukin-31 shared receptor. J Biol Chem 2006;281:36673-82. 